\title{
EXPERIMENTAL INVESTIGATION OF SNAP- THROUGH MOTION OF IN-PLANE MEMS SHALLOW ARCHES UNDER ELECTROSTATIC EXCITATION
}

\author{
Abdallah Ramini ${ }^{1}$, Mohammed L.F. Bellaredj ${ }^{1}$, Md Abdullah Al Hafiz ${ }^{1}$ and Mohammad I. Younis ${ }^{1,2, *}$ \\ ${ }^{1}$ Physical Sciences and Engineering Division, King Abdullah University of Science and Technology \\ Thuwal 23955-6900, Saudi Arabia. \\ ${ }^{2}$ Department of Mechanical Engineering, State University of New York at Binghamton, Binghamton, \\ NY 13902.
}

E-Mail: mohammad.younis@,kaust.edu.sa

\begin{abstract}
WE PRESENT AN EXPERIMENTAL INVESTIGATION FOR THE NONLINEAR DYNAMIC BEHAVIORS OF CLAMPED-CLAMPED IN-PLANE MEMS SHALLOW ARCHES WHEN EXCITED BY HARMONIC ELECTROSTATIC FORCES. FREQUENCY SWEEPS ARE CONDUCTED TO STUDY THE DYNAMIC BEHAVIORS IN THE NEIGHBORHOODS OF THE FIRST AND THIRD RESONANCE FREQUENCIES AS WELL AS THE SUPER HARMONIC RESONANCES. EXPERIMENTAL RESULTS SHOW LOCAL SOFTENING BEHAVIOR OF SMALL OSCILLATIONS AROUND THE FIRST RESONANCE FREQUENCY AND HARDENING BEHAVIOR AT THE THIRD RESONANCE FREQUENCY FOR SMALL DC AND AC LOADS. INTERESTING DYNAMIC SNAPTHROUGH CROSS-WELL MOTIONS ARE EVIDENTLY OBSERVED EXPERIMENTALLY AT HIGH VOLTAGES FOR THE FIRST TIME IN THE MICRO SCALE WORLD. IN ADDITION TO THE DYNAMIC SNAP-THROUGH MOTION, THE MEMS ARCH EXHIBITS LARGE OSCILLATIONS OF CONTINUOUS BAND OF SNAP-THROUGH MOTION BETWEEN THE SUPER HARMONIC RESONANCE REGIME AND THE FIRST PRIMARY RESONANCE REGIME. THIS CONTINUOUS BAND IS UNPRECEDENTED EXPERIMENTALLY IN THE MICRO/MACRO WORLD. THIS CONTINUOUS BAND OF SNAP-THROUGH MOTION CAN BE PROMISING FOR VARIETY OF SENSING, ACTUATION AND COMMUNICATIONS APPLICATIONS.
\end{abstract}

Keywords: In-plane MEMS, Shallow arch, Dynamic snap-through, Bistability, Nonlinear vibration.

\section{INTRODUCTION}

Bistable microstructures have drawn increasing attention of researchers working in the areas of microand nano-electromechanical systems (MEMS/NEMS) [1-15]. Bistability is one of the desirable characteristics for various applications, such as MEMS actuators, switches, relays, valves, clips, memory cells, and band pass filters. The most distinctive feature of bistable systems is the ability to remain in two different stable states at a single value of the actuation force. Other characteristics are the stability range and robustness, and high sensitivity to the structural geometry and residual stress. These characteristics have prompted the use of bistable systems. Also, small size bistable devices are rich in dynamics and represent a great stage for theoretical and experimental investigation of intriguing dynamic phenomena at the microscale. Latch-lock mechanisms [1], hinged multi segment mechanisms or compliant bistable mechanisms [2-5], residual compressive-stress buckled beams or pre-shaped stress-free curved beams [6-13], and double slider mechanisms [14-15] are the four main categories of bistable mechanisms that have been reported thus far. Saif [6] presented a tunable micromechanical bistable system that consists of a beam buckles along the transverse direction of two possible equilibrium states. Qui et al. [7] modeled and fabricated a mechanically-bistable mechanism called double curved beams using deep-reactive ion etching (DRIE). Charlot et al. [9] presented a buckled nanowire that can be actuated between two stable positions in order to use it as a non-volatile mechanical memory. They discussed the microfabrication process and analyzed mechanically and electrically the static buckling of nanowires. Michael and Kwok [10] studied theoretically and experimentally the bi-stability of a buckled multi-layered micro-bridge with elastically constrained boundary conditions. Casals-Terré et al. [16] presented an efficient bistable switch actuated dynamically at its resonance frequency. 
Due to their rich dynamics, there has been significant interest at the Macro scale in dynamics of arches and buckled beam configurations. Poon et al. [17] studied theoretically the snap-through phenomenon and the nonlinear dynamic response of a buckled clamped-clamped beam under harmonic excitation. They predicated small oscillations, large oscillations of continuous snap-through motion, and chaotic motion of irregular snap-through. Also they showed theoretically an unsteady continuous band of snapthrough between sub frequencies reaching to the primary resonance due to large exciting force and low damping ratio. Also, they reported switching from softening to hardening during the snap-through motion. Lee et al. [18] continued the previous study with an experimental and numerical investigation of the effect of the anti-symmetric mode shapes on the dynamic snap-through motion and the stability of the clamped-clamped buckled beam, especially when the resonance of the anti-symmetric mode is twice the resonance of the symmetric mode. Chandra et al. [19] examined the response of shallow arches of civil structures under sinusoidal distributed loading experimentally and numerically using finite-element analysis. They classified the regions of the dynamic behavior into a non-chaotic nonsnap-through, non-chaotic snap-through, chaotic non snap-through, and chaotic snap-through responses. Also, they [20] presented a numerical investigation using Poincare sections and basin of attraction plots of the dynamics shallow arches due to sinusoidal excitation and identified the regimes of the snap-through motion and no snap-through motions. In addition, they identified the forcing parameters that lead to snap-through, and eventually to chaotic response. Some researchers were trying to eliminate the snap-through motion. Stanciulescu et al. [21] used a nonlinear finite element model to eliminate the snap-through motion of an arch due a quasi-static thermomechanical load. Also, others concentrated on the nonlinear behaviors of curved beams by obtaining the periodic responses of the vibrations of isotropic/composite beams using a more efficient shooting technique [22].

While many structures were reported, initially curved clamped-clamped microbeam/shallow arches have recently emerged as a focus of intense studies [6-10, 23-43] and have gathered significant attention because of their geometric simplicity and broad applicability. Zhang et al. [23] concluded that the nonlinear dynamic instability behavior of the arch beam is due to the snap-through motion changing from the softening spring type to the hardening type. The snap-through and pull-in instabilities of the micro machined arch-shaped beams under an electrostatic loading were studied both theoretically and experimentally. Park and Hah [24] investigated analytically and experimentally the existence of the second stable state in a buckled-beam actuator that depends not only on the ratio of its initial rise to its thickness, but also on its residual stress. Krylov et al. [25] fabricated initially curved clamped-clamped microbeams and conducted theoretical and experimental investigation using optical and scanning electron microscopy on the static deflection. Li et al. [26] fabricated clamped-clamped composite structures and studied the nonlinear oscillations of curved cross-sectional MEMS resonators. Buchaillot et al. [27] presented and fabricated an out-of-plane clamped-clamped micro buckled beam. They forced the micro beam to vibrate by a harmonic force on the support and measured the transverse displacement using laser Doppler vibrometer. Roodenburg et al. [28] implemented a mechanical memory using clamped-clamped microbeams by buckling the beams to the snap-through in the post buckled state. The beams are buckled by passing a current (resistive heating) and generating a compressive stress. Das and Batra [29] solved the nonlinear partial differential equation governing of the arch numerically using Galerkin method to solve the static and transient dynamic problems due to DC voltage only. Krylov and Dick [30] presented theoretical snap-through and pull-in results of the transient nonlinear dynamics of an initially curved bistable micro beam actuated by combining the electrostatic and inertial forces. Krylov et al. [31] showed theoretically using lumped-parameter model for an initially curved or pre-buckled electrostatically actuated microbeams that switching is related with the dynamic snap-through and likely within interval of actuation voltages. Joe et al. [32] used a buckled silicon clamped-clamped mechanical resonators coated on one side to sense atmospheric chemical vapors. Their concept was based on using the critical bucking point to measure the change in frequency and transfer this change into mass sensing. Zhu and $\mathrm{Zu}$ [33] fabricated and tested a piezoelectric buckled microbeam to harvest energy from the snap-through motion by installing magnetic force at the midpoint. Medina et al. [34] studied and analyzed the symmetry breaking conditions of a shallow initially curved micro beam under a distributed electrostatic force. Also, Medina et al. [35] investigated theoretically and experimentally using image processing the symmetric and asymmetric buckling of a curved beam under axial and electrostatic force. Also, they experimentally investigated the stability and the snap-through motion of the initially curved microbeams. Sar and Pakdemirli [36] focused on the non-ideal boundary conditions and used the 
perturbation theory and the method of multiple scales to study the effect of these boundary conditions on a resonant curved microbeam actuated electrostatically.

Few works have focused on the dynamic response of arches under harmonic electrostatic loading. Younis and Ouakad [37] used the Galerkin method with five symmetric mode shapes of a straight beam to simulate the static and dynamic behavior of arches under AC and DC actuation. They showed various nonlinear behaviors, such as hysteresis, softening behavior, and dynamic pull-in. Ouakad and Younis [38] used a perturbation method to obtain analytically the forced vibration response of the arch due to DC and small AC loads and to show softening behavior. Also, they conducted numerical and experimental investigations on curved out of plane microbeams when excited by DC and AC loads showing softening and hardening behaviors near the first and third natural frequencies, respectively [39]. Ruzziconni et al. [40] added the axial force to their reduced-order model for an imperfect microbeam under an axial load and an electric excitation. Ouakad and Younis [41] investigated numerically and experimentally the dynamics of initially curved microbeams under electrodynamic forcing and the possibility of using their dynamic snap-through motion for filtering purposes. Alkharabsheh and Younis [42] investigated numerically using a shooting technique the dynamics of electrostatically actuated MEMS shallow arches with flexible (non-ideal) supports showing snapthrough motion and dynamic pull-in behavior. Also, they investigated numerically the effect of the axial force on MEMS shallow arches to tune their operating and their resonance frequencies and simulated an imperfect microbeam under an axial load and an electric excitation [43].

Most of the previous experimental works in MEMS arches have been for out of plane imperfect beams, which do not show dynamic snap-through motion. For in plane devices, where the arch can snapthrough dynamically without hitting the other electrode, only static snap-through has been studied experimentally. For the dynamic behavior, only theoretical works have been presented. In this work, we investigate for the first time experimentally the nonlinear dynamics of a clamped-clamped in-plane MEMS shallow arches when excited by harmonic electrostatic forces. We analyze and explore the dynamic behaviors of the in-plane motion of these shallow arches via frequency sweeps in the neighborhood of the first and third resonance frequencies. We also demonstrate the possibility of using the dynamic snap-through for band-pass filtering applications.

\section{Devices and experimental setup}

The in-plane MEMS shallow arches of this work have been fabricated by MEMCAP [44] from SOI wafers with highly conductive 25 um Si device layer. In this work, we consider two cases studies of beams of $600 \mu \mathrm{m}$ in length and $25 \mu \mathrm{m}$ in width, figure 1. The figure shows SEM images of the gap between the two electrodes $d=8.86 \mu \mathrm{m}$. Also, it shows the initial curvature $b_{o}=2.65 \mu \mathrm{m}$ that equals to the difference between the gap at the middle and the gap $d$ at the end of the arch. The two tested micro arches basically differ in their intrinsic axial residual stress. The rest of the arch dimensions are shown in table 1, as measured using an optical profilometer Zygo [45]. The error between the Zygo and SEM measurements is very small $\approx 2 \%$, therefore using the Zygo measurements have a very good accuracy in representing the dimensions of the arch. 


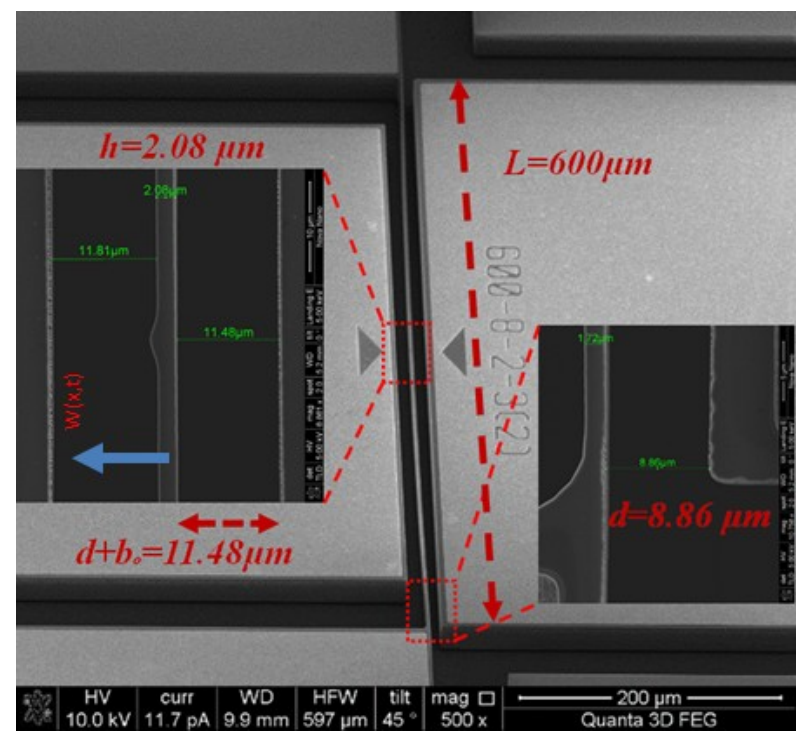

Figure 1. SEM images showing the top view of the in-plane MEMS shallow arch structure sandwiched between two pads with the dimensions of the gap and thickness at the middle and the end of the arch.

Table 1. The measured dimensipns of the test MEMS arches.

\begin{tabular}{llll} 
& $Z$ & $Z_{1}$ & SEM \\
\hline Gap (d) & $8.8 \mu \mathrm{m}$ & $8.7 \mu \mathrm{m}$ & $8.86 \mu \mathrm{m}$ \\
Thickness $(\mathrm{h})$ & $2 \mu \mathrm{m}$ & $2 \mu \mathrm{m}$ & $2.08 \mu \mathrm{m}$ \\
Initial curvature $\left(\mathrm{w}_{0}\right)$ & $2.7 \mu \mathrm{m}$ & $2.6 \mu \mathrm{m}$ & $2.62 \mu \mathrm{m}$
\end{tabular}
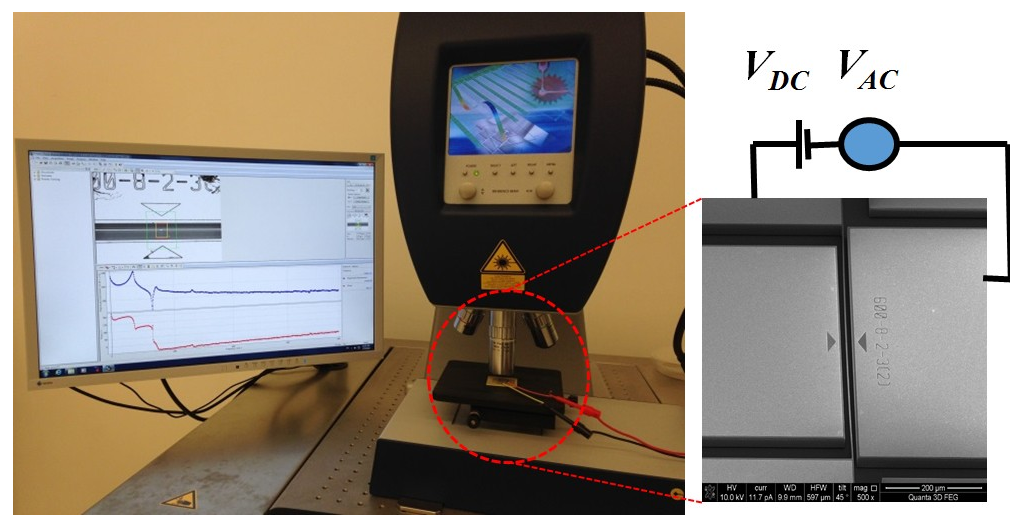

Figure 2. Experimental setup of MSA with the tested device.

For dynamic measurements and to conduct frequency sweeps, we use a Micro System Analyzer (MSA500) of Polytec [46] shown in figure 2, equipped with a stroboscopic video microscopy for in-plane motion analysis Planar Motion Analyzer (PMA-400) [46]. Using the PMA, we generate an amplified periodical sine signal to excite the in-plane arch. Due to the large gap separating each arch from the other stationary electrode, squeeze-film damping is not of significance influence here. Hence, these arches are tested in air.

\section{Problem Formulation}

The nonlinear equation governing the transverse motion $w(x, t)$ of the arch microbeam shown in figure 1 [38-43, 47-49] is as following

Where $x$ is the spatial position, $t$ is time and $w_{o}$ is the initial curvature of the arch. This arch has a Young's modulus $E$ and material density $\rho$ and is assumed to have a moment of inertia $I$. Also, it is subjected to a viscous damping coefficient $c$ and an axial stress $N$. This arch is subjected to an electrostatic force is composed of a DC component $V_{D C}$ superimposed to an AC harmonic load of amplitude $V_{A C}$ and a 
frequency $\Omega$. Also, $\varepsilon$ is the dielectric constant of air. The $\operatorname{arch}$ is of length $L$, of width $b$ and of thickness $h$.

Equation (2) presents the initial curvature of the arch where $b_{0}$ presents the initial rise at the middle of arch. Next, we solve the Eigen value problem to get the natural frequencies and mode shapes. Therefore, we solve the linearized undamped unforced equation (1) [47], which can be written as

Using the separation of variables where

where $\omega$ is the eigenvalue and $\varphi$ is the eigen-function. Then, we plug equation (4) into equation (3).

Substituting equation (4) in to equation (3) and separate the variables to get the spatial equation.

The spatial position solution has two parts: homogeneous part $\varphi_{h}(x)$ and the particular part $\varphi_{p}(x)$.

Substituting equation (7) in to equation (5) gives us the constant $c_{5}$ as function of the unknowns $A, B, C$ and $D$

Therefore, the Eigen function becomes

We are going to compare the experimental results from the FFT with the analytical results.

\section{Experimental Results}

4.1. Fast Fourier Transform (FFT) Measurements

At the beginning, we measure the linear resonance frequencies of the MEMS arches in air using ring down measurement and get the FFT. During the FFT measurement, the PMA generates multiple decaying signals to extract the resonance frequencies. Figure 3 shows the measured FFT. The first resonance frequency of Arch 1 is found at $70.7 \mathrm{kHz}$ corresponding to the first symmetric mode shape. The quality factor $(Q)$ can be calculated by

For the first mode shape, $Q=14.3$, resulting a damping ratio $\xi=1 / 2 Q=0.035$. For Arch 2, the first resonance frequency is at $86.6 \mathrm{kHz}$ and the quality factor is equal to 19.74 , which corresponds to a damping ratio $\xi=0.0253$. Hence, one can see that these arches are underdamped, which allows direct harmonic excitations in air.

(a)

Figure 3. FFT for the in-plane MEMS shallow arches. (a) Arch 1 has $f_{l}=70.7 \mathrm{kHz}$. (b) Arch 2 has $f_{l}=$ $86.6 \mathrm{kHz}$. 
In addition, the second resonance frequency is at $130.1 \mathrm{kHz}$ of Arch 1 correspondence to the first antisymmetric mode and the third resonance frequency is at $260.1 \mathrm{kHz}$, which is the second symmetric mode. To make sure that the second resonance frequency is for the anti-symmetric mode, we conducted a frequency sweep test using harmonic electrostatic forces and noticed that there is no measured inplane displacements around that frequency for both arches. All parameters, extracted from FFT measurements, are shown in table 2. Next, we compare the frequency values between the FFT measurements and the analytical Eigen-value problem in table 3. The difference between the experimentally measured and analytically simulated frequencies is coming from the axial residual stress.

Parameters from the FFT Measurements

\begin{tabular}{lcc}
\multicolumn{1}{c}{ Table 2. } & Arch-1 & Areh 2 \\
\hline$f_{1}(\mathrm{kHz})$ & 70.7 & 86.6 \\
$f_{3}(\mathrm{kHz})$ & 260.1 & 247 \\
$-3 d B$ for $f_{1}(\mathrm{~Hz})$ & 4945 & 4386.8 \\
$-3 d B$ for $f_{3}(\mathrm{kHz})$ & 49 & 55 \\
$Q$ of $1^{\text {st }}$ mode & 14.3 & 19.74 \\
$\xi$ of $1^{\text {st }}$ mode & 0.035 & 0.0253 \\
$Q$ of $3^{\text {rd }}$ mode & 5.31 & 4.5 \\
$\xi$ of $3^{\text {rd }}$ mode & 0.094 & 0.111
\end{tabular}

Table 3. Comparing the FFT measurements with numerical simulation for Arch 2

\begin{tabular}{lll} 
Table 3. & FFT Exprimal & Numerical simulation \\
& Measurements & No stres s \\
\hline$f_{1}(\mathrm{kHz})$ & 86.6 & 84.983 \\
$f_{3}(\mathrm{kHz})$ & 247 & 273
\end{tabular}

Our assumption here is that the squeeze-film damping is not dominant. In our case, the damping can be reasonably assumed to be viscous and linear. To verify this, we consider a simple incompressible model of squeeze-film damping of a long beam, which yields a damping coefficient of the form [49]

Where $\eta$ is the viscosity of the air equals $1.82 \times 10^{-5} \mathrm{~N} . \mathrm{s} / \mathrm{m}^{2}, k$ is the effective stiffness of the clampedclamped arch microbeam [49] and it is equal to

Using equations (13) and (12), the damping coefficient is found to be $c=2.45325 \times 10^{-7} \mathrm{~N} . \mathrm{s} / \mathrm{m}$. The corresponding damping ratio is $\zeta=0.343728 \times 10^{-3}$. Because the distance between the vibrating microstructure and the stationary electrode is very large $(d=8.86 \mu \mathrm{m})$; and squeeze film damping is proportional to $1 / d^{3}$; its influence in this case is very small. On the other hand, viscous air damping and other losses still can contribute to lowering the quality factor of this device; which requires other analytical models.

\subsection{The effect of DC and AC loads on the fundamental mode}

In this section, we perform forward frequency sweeps and show the frequency response curves of the MEMS arches due to a combined DC and AC harmonic amplitude loads near the primary resonance of the fundamental mode. Then, we extract the amplitude and phase for the frequency sweeps. As a first step, we show the dynamic frequency response curve for small values of DC and AC harmonic amplitude loads to obtain a linear dynamic behavior around the first resonance frequency for each arch as shown in figures $4 \mathrm{a}$ and $4 \mathrm{~b}$. Afterward, we increase the electrostatic voltage and notice the nonlinear behavior of these responses in figure 4. A softening behavior appears around the local attractor of the MEMS shallow arch [48]. The frequency response curves indicate that the quadratic nonlinearities of the initial curvature and electrostatic force are dominant over the geometric cubic nonlinearity causing a softening behavior of the frequency response curves. This fact has been investigated extensively in [38] using the perturbation method of multiple scales and a multi-modal expansion. To further illustrate this, we apply on equation (1) a first-mode approximation with the arch mode shape in the Galerkin 
procedure [49] and drop the damping, which results in the non-dimensional equation governing the modal amplitude $u$ of the first mode

Where $f$ is the projected modal force and $\omega_{o}=28.6, \alpha_{2}=8565.69$ and $\alpha_{3}=48595.6$. It turns out that this system with quadratic and cubic nonlinearities can exhibit softening or hardening behavior depending whether the quadratic nonlinearities are dominant over the cubic nonlinearities or not (full analysis of this can be found in [38]). According to [50], if the sign of the effective nonlinearity coefficient is positive, the behavior is hardening, otherwise the behavior is softening.

Based on equation (15), we found that $\alpha=-50909.5$, which proves that the inherent quadratic nonlinearity of the arch is dominant, and thus, it exhibits softening behavior regardless of the DC voltage.

Figure $4 \mathrm{~b}$ reveals also the super harmonic resonance of order two due to the quadratic nonlinearity and of order three due to the cubic nonlinearity. Figure 5 shows there is very small parasitic effects due to electrostatic charging of $\mathrm{Si}$ on the arch resonator, when $V_{D C}>V_{A C}$ or $V_{D C}<V_{A C}$. This figure shows frequency response sweeps for $V_{D C}=40 \mathrm{~V}$ and $V_{A C}=20 \mathrm{~V}$ and $V_{D C}=20 \mathrm{~V}$ and $V_{A C}=40 \mathrm{~V}$. The forcing term is the same in both cases and equals $\left(2 V_{D C} V_{A C}\right)$. But the effective DC terms $\left(V_{D C}{ }^{2}+0.5 V_{A C}{ }^{2}\right)$ are different in these cases. These effective DC terms affect the response, shifts the equilibrium position and lowers the frequency.

(a)

(b)

Figure 4. Experimental frequency forward sweep around the first symmetric mode shape (a) Arch 1.

(b) Arch 2 .

Figure 5. Experimental frequency forward sweep around the first symmetric mode shape for Arch 2 .

\subsection{The effect of $D C$ and $A C$ on the super harmonic resonances}

In figure $4 \mathrm{~b}$, we recognize that additional 2 frequencies emerged when Arch 2 is actuated by large electrostatic voltages. To determine if these frequencies are super harmonic resonances of order two and three due to the quadratic and cubic nonlinearities, we extract the time history at these frequencies in figure $6 \mathrm{a}$ and $6 \mathrm{~b}$ and display the phase portrait for each period in figure $6 \mathrm{c}$ and $6 \mathrm{~d}$. We found that the phase portrait has 3 loops at $26 \mathrm{kHz}$ in figure $6 \mathrm{c}$. This implies that this is a super harmonic resonance of order three. The phase portrait in Figure $6 \mathrm{~d}$ shows two loops at $35 \mathrm{kHz}$, which also confirms a super harmonic resonance of order two [48].

(a)

(c) (b)

(d)

Figure 6. Response of Arch 2 when excited by $\mathrm{V}_{\mathrm{DC}}=50 \mathrm{~V}$ and $\mathrm{V}_{\mathrm{AC}}=60 \mathrm{~V}$. (a) Time History at $26 \mathrm{kHz}$. (b) Time History at $35 \mathrm{kHz}$. (c) Phase portrait at $26 \mathrm{kHz}$. (d) Phase portrait at $35 \mathrm{kHz}$.

\subsection{The snap-through motion near the primary resonance of the first mode}

We further increase the electrostatic voltage and sweep the driving frequency to obtain the frequency response curves. Figures 7 and 8 depict the results for Arch 1. One can observe the emergence of a dynamic snap-through motion, where distinctive jumps to larger amplitude motion are reported near primary resonance. This dynamic snap-through motion indicates that the MEMS arch vibrate and jump from their local attractors to bigger global attractors [37-39, 49]. Also, the behavior indicates hardening oscillation. This hardening behavior is due to the geometric cubic nonlinearity of the structure, which becomes dominant over the quadratic nonlinearities [37-39, 49]. Beyond this band of frequencies, the arch gets back to the softening behavior. Moreover, the amplitude and the range of snap-through regime 
continue to increase with the increase of the excitation voltage. An intriguing potential use of this sudden jump in bistable arched MEMS structures is a band pass filter application, where the MEMS arch suddenly jumps to large amplitude over a narrow frequency range. In other words, it is a sudden change from a stop band to pass band. We also notice a coexistence behavior in figure 7 at $V_{D C}=40 \mathrm{~V}$ and $V_{A C}=60 \mathrm{~V}$, where the arch is switching back and forth between the vibration at large amplitude between the two attractors and the vibration at the first local attractor toward the end of the snapthrough motion.

Figure 7. Experimental frequency forward sweep showing dynamic snap-through motion for Arch 1.

Figure 8 shows the change in amplitude and phase between the regimes before and after snap-through. The amplitude indicates that the stiffness of the shallow arch increases during the snap-through band and then decreases after the snap-through band. There is a partial phase shift during snap-through band, and then the full phase shift of $180^{\circ}$ happens when the arch gets back to vibrate at the local attractor after the snap-through band.

Figure 8. Dynamic snap-through for Arch 1 excited by $V_{D C}=40 \mathrm{~V}$ and $\mathrm{V}_{\mathrm{AC}}=50 \mathrm{~V}$. (a) Amplitude (b) Phase shift.

(a)

(c) (d)

Figure 9. Experimental frequency forward sweep for Arch 2 showing dynamic snap-through motion and hysteresis (a) $\mathrm{V}_{\mathrm{DC}}=60 \mathrm{~V}$ and $\mathrm{V}_{\mathrm{AC}}=50 \mathrm{~V}$ (b) $\mathrm{V}_{\mathrm{DC}}=55 \mathrm{~V}$ and $\mathrm{V}_{\mathrm{AC}}=60 \mathrm{~V}$ (c) $\mathrm{V}_{\mathrm{DC}}=60 \mathrm{~V}$ and $\mathrm{V}_{\mathrm{AC}}=$ $55 \mathrm{~V}(\mathrm{~d}) \mathrm{V}_{\mathrm{DC}}=60 \mathrm{~V}$ and $\mathrm{V}_{\mathrm{AC}}=60 \mathrm{~V}$.

Next, we show the response of Arch 2 where we concentrate on sweeping the exciting frequency over a wide range. Figure 9 shows the response near the first primary resonance as well as near the super harmonic resonances. Figure 10 shows one snap shot of the response near the snap-through at the primary resonance. The figure shows the time history of the last cycle after reaching steady state and two images of the two bistable positions before and after the snap-through motion.

(a)

(b)

(c)

Figure 10. Arch 2 excited by $\mathrm{V}_{\mathrm{DC}}=60 \mathrm{~V}$ and $\mathrm{V}_{\mathrm{AC}}=60 \mathrm{~V}$ at $59 \mathrm{kHz}$ during the dynamic snapthrough motion (a) The time history of one cycle (b) The initial position before dynamic snap-through

(c) The final position snapping towards the lower electrode after dynamic snap-through.

Figures $9 \mathrm{c}$ and $9 \mathrm{~d}$ indicate the emergence of dynamic snap-through motion at the super harmonic regime. The sudden jump appears first in figure 9c and becomes like a continuous band when increasing the electrostatic voltages in figure $9 \mathrm{~d}$. To study this dynamic snap-through motion, we extracted the time history in figure 11a for the first jump noticed at $36 \mathrm{kHz}$ in figure $9 \mathrm{c}$. Also, we show the phase portrait from the measured displacement and velocity at $36 \mathrm{kHz}$ in figure $11 \mathrm{~b}$. These time history and phase portrait figures show that there are two frequencies as indicated by what seems to be two kinds of motion (almost two loops in the phase portrait).

(a)

(b)

Figure 11. Arch 2 excited by $\mathrm{V}_{\mathrm{DC}}=60 \mathrm{~V}$ and $\mathrm{V}_{\mathrm{AC}}=55 \mathrm{~V}$. (a) Time history of the one cycle at $36 \mathrm{kHz}$.

(b) The phase portrait at $36 \mathrm{kHz}$.

\subsection{Continuous snap-through band}

Next, we continue to increase the electrostatic forcing and study its effects on the dynamic snapthrough motion, figure 12. The small oscillations due to small voltages become large oscillations of continuous snap-through motion. These oscillations show up as continuous band of snap-through between super harmonic frequency regime reaching to the primary resonance regimes due to large 
exciting force, in agreement with the results reported for macro scale arches of Poon et al. [17]. These continuous bands start from $30 \mathrm{kHz}$ up to $65 \mathrm{kHz}$. At the beginning of this band, the arch behaves nonlinearly and switches between softening to hardening back and forth around super harmonic frequencies. Eventually, this continuous band behavior might lead chaotic motion of irregular snapthrough when further increasing the driving electrostatic force [17].

(a)

(b)

Figure 12. Experimental frequency forward sweep showing continuous band of dynamic snap-through motion for Arch 2 (a) $\mathrm{V}_{\mathrm{DC}}=60 \mathrm{~V}$ and $\mathrm{V}_{\mathrm{AC}}=70 \mathrm{~V}$ (b) $\mathrm{V}_{\mathrm{DC}}=65 \mathrm{~V}$ and $\mathrm{V}_{\mathrm{AC}}=60 \mathrm{~V}$.

\subsection{The hardening behavior of the third mode}

Next, we study the dynamic behaviors and obtain the frequency response curves near the third primary resonances in figure 13. The results show that the responses are almost linear for smaller electrostatic excitations. But for large excitation forces, the arches behave nonlinearly showing hardening behavior. Because of the high damping, due to operating in air, and the limitations in the maximum amplitude of the electrostatic voltage at that frequency range, we were unable to excite the MEMS arches for amplitude beyond $1 \mu \mathrm{m}$.

(b)

Figure 13. Experimental frequency forward sweep around the third mode shape (a) Arch 1. (b) Arch 2.

\section{Summary and Conclusions}

We investigated experimentally the dynamic behavior of an electrostatically actuated clamped-clamped in-plane MEMS shallow arch near the first and third primary resonances, and the super harmonic resonances using frequency sweeps. Most importantly, the experimental results brings out various characteristic and dynamic features such as: (a) small oscillations showing softening behavior for low excitation forces, (b) large oscillations of dynamic snap-through between the two stable state, for the first time experimentally, showing hardening behavior and (c) large oscillations of continuous band of dynamic snap-through motion. This experimentally continuous band of snap-through motion is a novel dynamic behavior in the micro/macro range. Also, these results show nonlinear dynamic instability behavior of the beam, changing from the softening spring type to the hardening type due to the snapthrough motion. Moreover, we showed some kind of hysteresis and coexistence of attractors where the arch is switching back and forth between the vibration at large amplitude between the two attractors and the vibration at the first local attractor at the beginning and the end of the snap-through regime. Furthermore, we showed a hardening effect near the primary resonance of the third mode.

The dynamic snap-through band can be utilized to fabricate more sensitive and noise repelling band pass filters in the micro and nano ranges. There is no force or amplitude outside the snap-through regime compared to the on-state during the snap-through regime of the arch, these reasons result in minimal power consumption, and stop band tempering. Also, this interesting jump has a great potential for designing MEMS switches, actuators, mechanical memories, micro-relays and band pass filters that are sensitive and resilient to noise. Also, it could be used for energy harvesters because the strong nonlinearity is coming from the initial curvature and not from the electrostatic forcing. Hence, large amplitude motion, and hence significant power, can be harvested from the ambient if these arches can be driven to snap-through motion from the environment.

\section{Acknowledgment}

This work has been supported by KAUST.

\section{REFERENCES}

[1] Hoffmann M, Kopka P, Voges E. All-silicon bistable micromechanical fiber switch based on advanced bulk micromachining. Selected Topics in Quantum Electronics, IEEE Journal of. 1999;5(1):46-51.

[2] Masters ND, Howell LL. A self-retracting fully compliant bistable micromechanism. Microelectromechanical Systems, Journal of. 2003;12(3):273-80. 
[3] Hwang $\mathrm{I}-\mathrm{H}$, Shim $\mathrm{Y}-\mathrm{S}$, Lee $\mathrm{J}-\mathrm{H}$. Modeling and experimental characterization of the chevron-type bi-stable microactuator. Journal of Micromechanics and Microengineering. 2003;13(6):948.

[4] Ko JS, Lee MG, Han JS, Go JS, Shin B, Lee D-S. A laterally-driven bistable electromagnetic microrelay. ETRI journal. 2006;28(3):389-92.

[5] Receveur RA, Marxer CR, Woering R, Larik VC, De Rooij N-F. Laterally moving bistable MEMS DC switch for biomedical applications. Microelectromechanical Systems, Journal of. 2005;14(5):1089-98.

[6] Saif MTA. On a tunable bistable MEMS-theory and experiment. Microelectromechanical Systems, Journal of. 2000;9(2):157-70.

[7] Qiu J, Lang JH, Slocum AH. A curved-beam bistable mechanism. Microelectromechanical Systems, Journal of. 2004;13(2):137-46.

[8] Hälg B. On a micro-electro-mechanical nonvolatile memory cell. Electron Devices, IEEE Transactions on. 1990;37(10):2230-6.

[9] Charlot B, Sun W, Yamashita K, Fujita H, Toshiyoshi H. Bistable nanowire for micromechanical memory. Journal of Micromechanics and Microengineering. 2008;18(4):045005.

[10] Michael A, Kwok CY. Design criteria for bi-stable behavior in a buckled multi-layered MEMS bridge. Journal of Micromechanics and microengineering. 2006;16(10):2034.

[11] Cabal A, Ross D, editors. Snap-through bilayer microbeam. Proc MSM; 2002.

[12] Sulfridge M, Saif T, Miller N, Meinhart M. Nonlinear dynamic study of a bistable MEMS: model and experiment. Microelectromechanical Systems, Journal of. 2004;13(5):725-31.

[13] Rossiter J, Stoimenov B, Mukai T, editors. A self-switching bistable artificial muscle actuator. SICE-ICASE, 2006 International Joint Conference; 2006: IEEE.

[14] Jensen BD, Howell LL. Bistable configurations of compliant mechanisms modeled using four links and translational joints. Journal of Mechanical Design. 2004;126(4):657-66.

[15] Tsay J, Su L-Q, Sung C-K. Design of a linear micro-feeding system featuring bistable mechanisms. Journal of Micromechanics and Microengineering. 2005;15(1):63.

[16] Casals-Terré J, Fargas-Marques A, Shkel A M. Snap-action bistable micromechanisms actuated by nonlinear resonance, Journal Of Microelectromechanical Systems. 2008;17(5): 1082-1093.

[17] Poon W, Ng C, Lee Y. Dynamic stability of a curved beam under sinusoidal loading. Proceedings of the Institution of Mechanical Engineers, Part G: Journal of Aerospace Engineering. 2002;216(4):209-17.

[18] Lee $\mathrm{Y}$, Poon $\mathrm{W}, \mathrm{Ng} \mathrm{C}$. Anti-symmetric mode vibration of a curved beam subject to autoparametric excitation. Journal of Sound and Vibration. 2006;290(1):48-64.

[19] Chandra Y, Wiebe R, Stanciulescu I, Virgin LN, Spottswood SM, Eason TG. Characterizing dynamic transitions associated with snap-through of clamped shallow arches. Journal of Sound and Vibration. 2013;332(22):5837-55.

[20] Chandra Y, Stanciulescu I, Virgin LN, Eason TG, Spottswood SM. A numerical investigation of snap-through in a shallow arch-like model. Journal of Sound and Vibration. 2013;332(10):2532-48.

[21] Stanciulescu I, Mitchell T, Chandra Y, Eason T, Spottswood M. A lower bound on snap-through instability of curved beams under thermomechanical loads. International Journal of Nonlinear Mechanics. 2012;47(5):561-75.

[22] Ibrahim S, Patel B, Nath Y. Modified shooting approach to the nonlinear periodic forced response of isotropic/composite curved beams. International Journal of Nonlinear Mechanics. 2009;44(10):1073-84.

[23] Zhang Y, Wang Y, Li Z, Huang Y, Li D. Snap-through and pull-in instabilities of an arch-shaped beam under an electrostatic loading. Microelectromechanical Systems, Journal of. 2007;16(3):684-93.

[24] Park S, Hah D. Pre-shaped buckled-beam actuators: Theory and experiments. Sensors and Actuators A: Physical. 2008;148(1):186-92.

[25] Krylov S, Ilic BR, Schreiber D, Seretensky S, Craighead H. The pull-in behavior of electrostatically actuated bistable microstructures. Journal of Micromechanics and Microengineering. 2008;18(5):055026.

[26] Li H, Piekarski B, DeVoe D, Balachandran B. Nonlinear oscillations of piezoelectric microresonators with curved cross-sections. Sensors and Actuators A: Physical. 2008;144(1):194-200.

[27] Buchaillot L, Millet O, Quévy E, Collard D. Post-buckling dynamic behavior of selfassembled 3D microstructures, Microsystem Technologies. 2008; 14(1): 69-78. 
[28] Roodenburg D, Spronck J, Van der Zant H, Venstra W. Buckling beam micromechanical memory with on-chip readout. Applied Physics Letters. 2009;94(18):183501.

[29] Das K, Batra R C. Symmetry breaking, snap-through and pull-in instabilities under dynamic loading of microelectromechanical shallow arches. Smart Materials and Structures. 2009; 18(11): 115008.

[30] Krylov S, Dick N. Pull-in Dynamics of Electrostatically Actuated Bistable Micro Beams. Advanced Materials and Technologies for Micro/Nano-Devices, Sensors and Actuators: Springer; 2010. p. 117-28.

[31] Krylov S, Ilic BR, Lulinsky S. Bistability of curved microbeams actuated by fringing electrostatic fields. Nonlinear Dynamics. 2011;66(3):403-26.

[32] Joe J, Linzon Y, Adiga V, Barton R, Kim M, Ilic B, Krylov S, Parpia J, Craighead H. Stress-based resonant volatile gas microsensor operated near the critically buckled state. Journal of Applied Physics. 2012; 111: 104517.

[33] Zhu Y, Zu JW. Enhanced buckled-beam piezoelectric energy harvesting using midpoint magnetic force. Applied Physics Letters. 2013;103(4):041905.

[34] Medina L, Gilat R, Krylov S. Symmetry breaking in an initially curved micro beam loaded by a distributed electrostatic force. International Journal of Solids and Structures. 2012;49(13):1864-76.

[35] Medina L, Gilat R, llic B, Krylov S. Experimental investigation of the snap-through buckling of electrostatically actuated initially curved pre-stressed micro beams. Sensors and Actuators A: Physical. 2014;220:323-32.

[36] Sar G, Pakdemirli M. Effects of non-ideal boundary conditions on the vibrations of a slightly curved micro beam. 9TH INTERNATIONAL CONFERENCE ON MATHEMATICAL PROBLEMS IN ENGINEERING, AEROSPACE AND SCIENCES: ICNPAA 2012; 2012: AIP Publishing.

[37] Younis M, Ouakad HM, Alsaleem FM, Miles R, Cui W. Nonlinear dynamics of MEMS arches under harmonic electrostatic actuation. Microelectromechanical Systems, Journal of. 2010;19(3):647-56.

[38] Ouakad HM, Younis MI. The dynamic behavior of MEMS arch resonators actuated electrically. International Journal of Nonlinear Mechanics. 2010;45(7):704-13.

[39] Ouakad HM, Younis MI. On using the dynamic snap-through motion of MEMS initially curved microbeams for filtering applications. Journal of Sound and Vibration. 2014;333(2):555-68.

[40] Ruzziconi L, Younis MI, Lenci S. An efficient reduced-order model to investigate the behavior of an imperfect microbeam under axial load and electric excitation. Journal of Computational and Nonlinear Dynamics. 2013;8(1):011014.

[41] Ruzziconi L, Lenci S, Younis MI. An imperfect microbeam under an axial load and electric excitation: nonlinear phenomena and dynamical integrity. International Journal of Bifurcation and Chaos. 2013;23(02):1350026.

[42] Alkharabsheh S, Younis M. Dynamics of MEMS arches of flexible supports. Journal of Microelectromechanical Systems. 2013;22(1):216-24.

[43] Alkharabsheh SA, Younis MI. Statics and dynamics of mems arches under axial forces. Journal of Vibration and Acoustics. 2013;135(2):021007.

[44] MEMSCAP, http://www.memscap.com

[45] Zygo Corporation, http://www.zygo.com/

[46] Polytec Inc., http://www.polytec.com/us/

[47] Nayfeh A, Kreider W, Anderson T. Investigation of natural frequencies and mode shapes of buckled beams. AIAA Journal. 1995; 33(6): 1121-1126.

[48] Bataineh A, Younis $M$, Dynamics of an Imperfect Microbeam Considering its Exact Shape. Microsystem Technologies 2014:doi 10.1007/s00542-014-2349-7.

[49] Younis MI. MEMS linear and nonlinear statics and dynamics: Springer Science \& Business Media; 2011.

[50] Nayfeh AH and Mook DT. Nonlinear Oscillations, Wiley, New York, 1995. 